\title{
LETTERS
}

DOI 10.1002/art.41702

\section{COVID-19 disease in patients with recurrent pericarditis during treatment with anakinra: comment on the article by Navarro-Millán et al}

To the Editor:

We read with interest the article by Dr. Navarro-Millán and colleagues about the use of anakinra to prevent mechanical ventilation in patients with COVID-19 (1). However, it is also important to consider patients who develop COVID-19 while being treated with anakinra for their underlying condition (2).

We describe 5 patients, median age 43 years, with recurrent pericarditis (post-pericardiotomy in 1 case; idiopathic pericarditis in 4 cases) who developed COVID-19 disease during treatment with anakinra. Median duration of recurrent pericarditis was 48 months. All patients were being treated with anakinra when COVID-19 disease occurred, after having initially received treatment with glucocorticoids and/or nonsteroidal antiinflammatory drugs (including colchicine) (Table 1).

The patients developed COVID-19 disease between March 2020 and October 2020. Symptoms, usually mild, included fever, cough, ageusia, anosmia, headache, diarrhea, dyspnea, and chest pain (Table 1). SARS-CoV-2 was diagnosed by nasopharyngeal swab in 4 patients, and by serologic test in 1 patient, after symptoms began. Two patients went to the emergency room; in one case, chest radiograph showed a small lung infiltrate, but neither of the patients required hospitalization. Treatment with anakinra was continued unchanged, and 3 patients received additional therapies after the development of COVID-19 disease (Table 1). All patients recovered completely within 15 days and had no recurrence of pericarditis.

Polytherapy is often necessary in patients with recurrent pericarditis and treatment with an interleukin-1 receptor antagonist may lead to resolution of symptoms (3); however, a concern may be raised that biologic therapy could aggravate the clinical course of COVID-19. Our small case series shows that anakinra therapy in patients with recurrent pericarditis may be associated with a benign clinical course. We propose that there is no reason to discontinue anakinra therapy if a patient with recurrent pericarditis develops COVID-19 disease (4-7). Our recommendation is consistent with the findings obtained in the study by Dr. Navarro-Millán et al (1).

Dr. Brucato has received research support from Sobi and Acarpia. Dr. Imazio has received consulting fees or honoraria from Kiniksa and Sobi (less than $\$ 10,000$ each).
Enrica Negro, MD iD
Lucia Trotta, MD
Massimo Pancrazi, MD
Emanuele Bizzi, MD
Martino Brenna, MD
Fatebenefratelli Hospital
Milan, Italy

Table 1. Summary of main features of patients *

\begin{tabular}{|c|c|c|c|c|c|c|}
\hline $\begin{array}{l}\text { Patient/ } \\
\text { age/sex }\end{array}$ & $\begin{array}{l}\text { Pericardial } \\
\text { disease } \\
\text { duration, } \\
\text { months }\end{array}$ & $\begin{array}{l}\text { Therapy when } \\
\text { cOVID-19 occurred }\end{array}$ & $\begin{array}{c}\text { COVID-19 } \\
\text { clinical features }\end{array}$ & $\begin{array}{c}\text { Adjusted/additional } \\
\text { therapies during } \\
\text { COVID-19 }\end{array}$ & $\begin{array}{l}\text { Hospitalization } \\
\text { or ER visit }\end{array}$ & $\begin{array}{l}\text { Duration of } \\
\text { COVID-19 } \\
\text { symptoms, } \\
\text { days }\end{array}$ \\
\hline $1 / 54 / M$ & 12 & $\begin{array}{l}\text { Anakinra (100 mg every } \\
48 \text { hours) }\end{array}$ & $\begin{array}{l}\text { Fever; cough; infiltrate in } \\
\text { right middle lobe on } \\
\text { chest radiograph; CRP } \\
\text { and D-dimer elevation }\end{array}$ & Azithromycin & ER visit & 5 \\
\hline 2/15/M & 21 & $\begin{array}{l}\text { Anakinra (100 mg every } \\
3 \text { days); colchicine (1 mg/day) }\end{array}$ & Low-grade fever; asthenia & None & None & 2 \\
\hline $3 / 43 / F$ & 48 & $\begin{array}{l}\text { Anakinra (100 mg every } \\
4 \text { days); colchicine (1 mg/day) }\end{array}$ & $\begin{array}{l}\text { Fever; cough for } 4 \text { days; } \\
\text { ageusia; anosmia; } \\
\text { diarrhea; headache }\end{array}$ & None & None & 15 \\
\hline $4 / 35 / F$ & 54 & $\begin{array}{l}\text { Anakinra (100 mg/day); } \\
\text { colchicine (1.5 mg/day); } \\
\text { nadolol }\end{array}$ & $\begin{array}{l}\text { Dry cough; fever for } 3 \text { days; } \\
\text { asthenia; diarrhea; chest } \\
\text { pain; normal CRP }\end{array}$ & $\begin{array}{l}\text { Prednisone ( } 25 \mathrm{mg} / \text { day } \\
\text { for } 5 \text { days) then } \\
12.5 \mathrm{mg} / \text { day); } \\
\text { indomethacin }\end{array}$ & ER visit & 10 \\
\hline $5 / 78 / F$ & 60 & $\begin{array}{l}\text { Anakinra (100 mg/day); } \\
\text { colchicine (1 mg/day); } \\
\text { prednisone ( } 2.5 \mathrm{mg} \\
\text { every } 2 \text { days) }\end{array}$ & $\begin{array}{l}\text { Low-grade fever for } 2 \text { days; } \\
\text { dyspnea }\end{array}$ & $\begin{array}{l}\text { Prednisone (2.5 mg/day); } \\
\text { acetaminophen; } \\
\text { amoxicillin-clavulanic } \\
\text { acid }\end{array}$ & None & 15 \\
\hline
\end{tabular}

* $\mathrm{ER}$ = emergency room; CRP = C-reactive protein. 


\author{
Vartan Mardigyan, MD \\ McGill University \\ and Jewish General Hospital \\ Montreal, Quebec, Canada \\ Massimo Imazio, MD iD \\ Azienda Ospedaliero Universitaria Città della Salute e della \\ Scienza di Torino \\ Turin, Italy \\ Antonio Brucato, MD iD \\ Università di Milano \\ and Fatebenefratelli Hospital \\ Milan, Italy
}

1. Navarro-Millán I, Sattui SE, Lakhanpal A, Zisa D, Siegel CH, Crow MK. Use of anakinra to prevent mechanical ventilation in severe COVID-19: a case series. Arthritis Rheumatol 2020;72:1990-7.

2. Imazio M, Brucato A, Lazaros G, Andreis A, Scarsi M, Klein A, et al. Anti-inflammatory therapies for pericardial diseases in the COVID-19 pandemic: safety and potentiality [review]. J Cardiovasc Med (Hagerstown) 2020;21:625-9.
3. Klein AL, Imazio M, Brucato A, Abbate A, Fang F, Insalaco A, et al. Phase 3 trial of interleukin-1 trap rilonacept in recurrent pericarditis. N Engl J Med 2020;2021;384:31-41.

4. Putman M, Chock YP, Tam H, Kim AH, Sattui SE, Berenbaum F, et al. Antirheumatic disease therapies for the treatment of COVID-19: a systematic review and meta-analysis. Arthritis Rheumatol 2021;73:36-47.

5. Chau AS, Weber AG, Maria NI, Narain S, Liu A, Hajizadeh N, et al. The longitudinal immune response to coronavirus disease 2019: chasing the cytokine storm [review]. Arthritis Rheumatol 2021; 73:23-35

6. Scarsi M, Piantoni S, Colombo E, Airó P, Richini D, Miclini M, et al. Association between treatment with colchicine and improved survival in a single-centre cohort of adult hospitalised patients with COVID-19 pneumonia and acute respiratory distress syndrome. Ann Rheum Dis 2020;79:1286-9.

7. Aomar-Millán A, Salvatierra J, Torres-Parejo Ú, Faro-Miguez N, Callejas-Rubio JL, Ceballos-Torres Á, et al. Anakinra after treatment with corticosteroids alone or with tocilizumab in patients with severe COVID-19 pneumonia and moderate hyperinflammation: a retrospective cohort study. Intern Emerg Med 2021;16:843-52. 